\title{
BMJ Open Which factors determine treatment choices in patients with advanced kidney failure? a protocol for a co- productive, mixed methods study
}

\author{
Gareth Roberts (D) , ${ }^{1}$ James A Chess, ${ }^{2}$ Teri Howells, ${ }^{3}$ Leah Mc Laughlin, ${ }^{4}$ \\ Gail Williams, ${ }^{5}$ Joanna M Charles, ${ }^{6}$ D J Dallimore (D , ${ }^{7}$ Rhiannon Tudor Edwards, ${ }^{6}$ \\ Jane Noyes (i) ${ }^{4}$
}

To cite: Roberts G, Chess JA, Howells $\mathrm{T}$, et al. Which factors determine treatment choices in patients with advanced kidney failure? a protocol for a co-productive, mixed methods study. BMJ Open 2019;9:e031515. doi:10.1136/ bmjopen-2019-031515

- Prepublication history for this paper is available online. To view these files, please visit the journal online (http://dx.doi org/10.1136/bmjopen-2019031515).

Received 30 May 2019 Revised 12 August 2019 Accepted 28 August 2019
Check for updates

(C) Author(s) (or their employer(s)) 2019. Re-use permitted under CC BY-NC. No commercial re-use. See rights and permissions. Published by BMJ.

For numbered affiliations see end of article.

Correspondence to

Dr Gareth Roberts;

gareth.roberts7@wales.nhs.uk

\section{ABSTRACT}

Introduction Kidney disease is common, affecting up to 1 in 10 of the adult population, and the numbers are expected to rise over the next decade. There are three main treatments that are available to patients with kidney disease: transplantation, dialysis and supportive care without dialysis. Dialysis can occur in a dialysis unit or in a person's home, but unit-based dialysis remains the most common initial treatment for patients in Wales. This is a cause for concern as most studies suggest that it is associated with the lowest quality of life and the highest mortality, and is a more expensive treatment option. This study aims to identify the factors that lead to patients choosing unit-based haemodialysis rather than homebased dialysis with a view to informing future changes in patient education and service commissioning in Wales. A secondary aim is to determine if the co-production of research leads to more sustainable services.

Methods and analysis This mixed-method study taking place between October 2018 and September 2020 will use a sequential explanatory design whereby the descriptive quantitative cross-sectional analysis of linked health and administrative data sets inform qualitative data collection from patients, carers and health and care professionals. Qualitative findings will be used to interpret or explain quantitative descriptive results. Additional strands to the study include a review of materials and education provided to patients and an economic review of treatment modalities.

Ethics and dissemination The study will be conducted in accordance with the principles expressed in the Declaration of Helsinki. It has full approval from Health and Care Research Wales Research Ethics Committee \#5. As a co-productive study involving patients, clinicians, third sector partners and academics, findings from this study will be shared on a continual basis. Study results will be published in peer-reviewed journals and presented at national and international conferences.

\section{INTRODUCTION}

It is estimated that the total cost of chronic kidney disease (CKD), in England in 2009 to 2010 was between $£ 1.44$ and $£ 1.45$ billion, which equates to approximately $1.3 \%$ of all
Strengths and limitationsof this study

- Principles of co-production mean that in this study National Health Service staff, patients, carers and other organisations will be working with academics to undertake research.

- The mixed-methods sequential explanatory design employed in this study is likely to provide both breadth and depth to our understanding of dialysis choices.

- The small sample for the qualitative study will not capture all aspects of patient choice.

- Wales is a small country (population 3 million) and healthcare is a fully devolved area of policy, which provides a manageable closed-system for study, and findings can rapidly change practice and improve patients care.

- There is a limited contemporary economic evidence base of kidney dialysis; therefore, this study addresses a knowledge gap in the literature.

National Health Service (NHS) spending in that year. ${ }^{12}$ The National Institute for Health and Care Excellence ${ }^{3}$ estimated approximately 7000 excess strokes and 12000 excess myocardial infarctions occurred in people with CKD in 2009 to 2010 (relative to an age-matched and gender-matched population without chronic kidney disease), with an estimated cost of between $£ 174$ and $£ 178$ million. ${ }^{2}$

Incidence and prevalence of dialysis is higher in Wales than in other parts of the UK and despite an estimated $30 \%$ of patients being suitable for home therapies ${ }^{4}$ only $15 \%$ in Wales receive peritoneal dialysis and only $6.9 \%$ home haemodialysis ${ }^{5}$ with significant variation in patient modality choice across the country. Previous systematic reviews ${ }^{6} 7$ and single centre studies ${ }^{89}$ have identified a number of potentially modifiable factors that influence patient choice. These include peer 
influence, timing of information, treatment flexibility, quality of life and a desire to maintain the status quo. Though of interest, the findings of these studies may not be directly applicable to pre-dialysis patients in Wales. Of note, none of the studies were based in the UK, and only one study focused on pre-dialysis patients. Other countries have significantly different healthcare systems to the UK, with different geographical constraints and different financial incentives/disincentives for home therapies as well as different modes of delivering pre-dialysis education.

The most comprehensive qualitative study of decision-making was recently undertaken in New Zealand $(\mathrm{NZ})^{89}$ and concluded that the level of support available and socioeconomic status were key determinants of whether patients chose a home therapy. It is not clear whether the same situation applies in Wales since NZ is unique in terms of the number of patients dialysing at home $(50 \%$ vs $22 \%$ in Wales). Of note, it was found that ethnicity and low household income was associated with lower preferences for a home therapy. However, patients in $\mathrm{NZ}$ choosing home therapies suffer out of pocket costs which is not the case in Wales. ${ }^{10}$

\section{Rationale}

None of the studies reviewed ${ }^{810-15}$ have combined clinical and socio-demographic data collection with qualitative study. Thus far, no studies have been undertaken in collaboration with service commissioners with the prespecified aim of bringing about optimal outcomes in patients by understanding their current choice of treatment, so that optimal treatment decisions are made in the future. We feel there is a pressing need for this, and in Wales we have a unique opportunity to collaborate with the service commissioners to both inform and directly influence patient care.

Both The National Institute for Clinical Excellence (NICE) and the Welsh Renal Clinical Network (WRCN who commission renal services in Wales) have recognised that too many patients are starting dialysis via unit-based haemodialysis (UHD). Given the current financial crisis that the NHS is facing, it is particularly worrying that the least cost-effective and worst therapy in terms of quality of life (QOL) remains the the most common choice in Wales. NICE have published two separate reports outlining the potential benefits of using home therapies. ${ }^{16} 17$ The NICE report on home dialysis suggested that up to $15 \%$ of all haemodialysis patients in the UK would be suitable for home-based haemodialysis while the NICE costing review of peritoneal dialysis (PD) suggested that up to $50 \%$ of patients would be suitable for PD. Home dialysis (and particularly short, daily home dialysis) has been shown to improve both mortality and quality of life, with observational studies and one randomised trial finding that more frequent dialysis improves outcomes. ${ }^{18}$ NICE and the commissioners of the service in Wales (WRCN) therefore agree that we need to increase the number of patients opting for a home therapy rather than UHD.
More recently, the WRCN published its delivery plan for 2016 to 2020. The renal network emphasise the need to make home therapies available to as many patients as possible who cannot have a pre-emptive transplant. Of note, the network strategy highlights that UHD should be a default option only when pre-emptive transplant and home therapies have been actively excluded.

As well as improving patient outcomes, reducing the number of patients on UHD may confer an economic benefit. This again has been highlighted by NICE, with an estimated saving for NHS England of £20 million per annum if home therapy was optimised. ${ }^{17}$ Even a more conservative estimate of $1 \%$ in the number of PD patients was estimated to save $£ 4$ million over 5 years.

\section{Project description}

This is a 2 year mixed method co-productive study integrating analysis of the NHS electronic renal patient records database, documentary analysis of the current education programme in each renal unit, qualitative interviews with patients, unpaid carers and professionals, and health economics rapid review and analysis (see figure 1).

\section{Aims and objectives}

The aim of the study is to develop a better understanding of the factors that impact treatment choices across Wales, and specifically to delineate why so many patients default to UHD rather than choosing therapies that offer better survival, better QOL and deliver better public value.

A secondary aim is to look at the sustainability of current and proposed alternative service provision.

1. To investigate the factors that impact on the dialysis choices made by patients with CKD in Wales.

2. To gain a comprehensive understanding of current pre-dialysis education across Wales to highlight modifiable factors that might be addressed.

3 . To assess the current cost to the NHS of dialysis treatment options.

4. To assess the efficacy of co-productive research in service improvement.

A list of the research questions are set out in figure 2 .

\section{METHODS AND ANALYSIS Theoretical framework}

This study is informed by a theoretical framework contained within the Making Good Decisions in Collaboration (MAGIC) shared patient decision-making intervention. The programme was developed for the UK NHS in 2010 designed to test and identify the best ways to embed shared decision-making into routine primary and secondary care using quality improvement methods. ${ }^{19}$ While many clinicians feel that they already involve patients in decisions about their care, shared decision-making differs from usual practice by building relationships through the clinical encounter so that information is shared and patients are supported to deliberate 


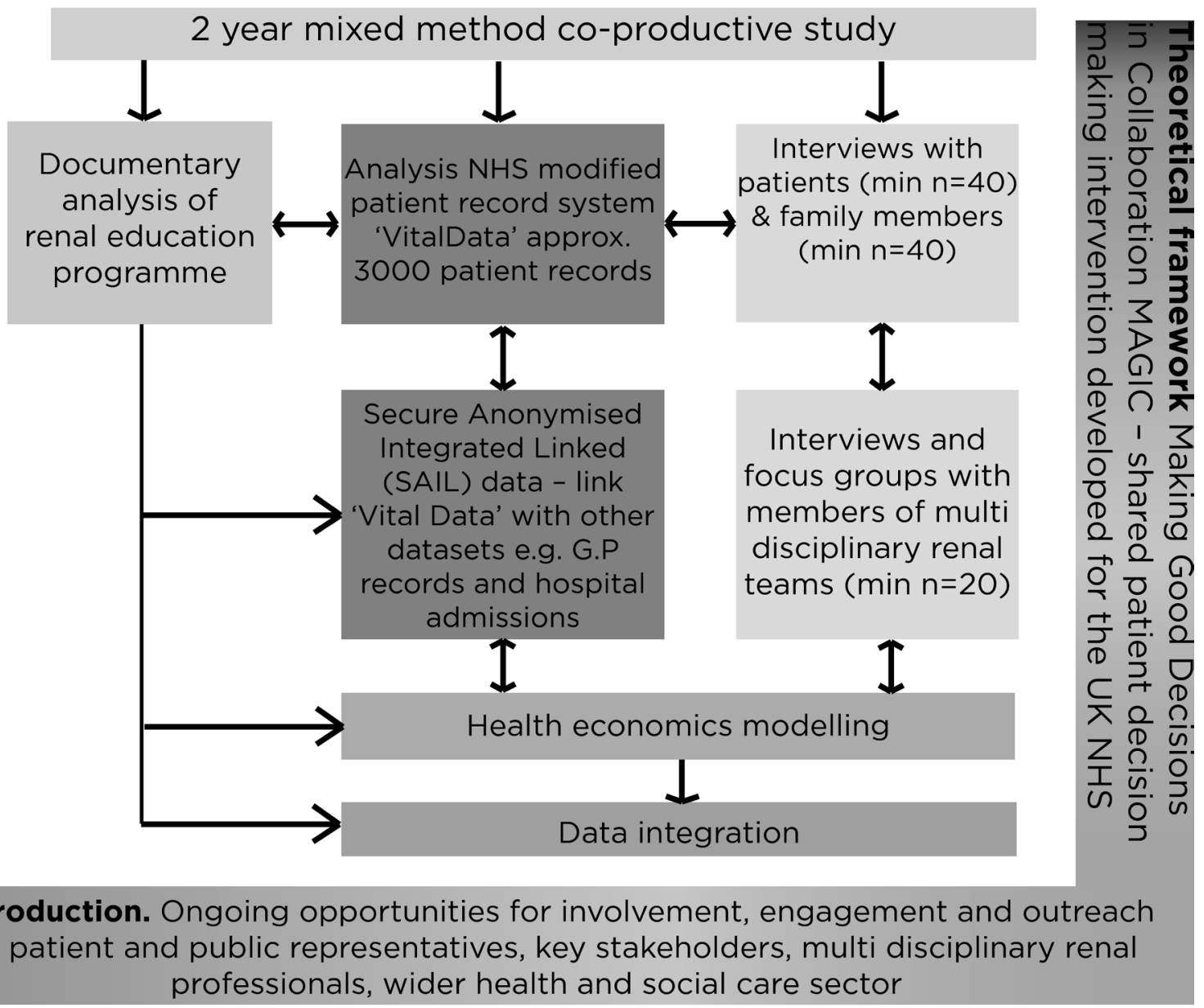

Figure 1 Study design. GP, general practitioner; NHS, National Health Service.

and express their preferences and views during the decision-making process. ${ }^{20}$ The MAGIC model (figure 3) provides a path to shared decision-making which is based on choice, option and decision talk underpinning the research aims of this study.

The most fundamental learning point from evaluations of the MAGIC programme ${ }^{21}$ is that no single intervention will succeed in isolation and that effective shared decision-making requires a range of interventions working together holistically across healthcare settings.

\section{Methods}

In summary, there are five strands to this study including co-production as an overarching process. The cohort for all elements of the study however, will be taken from the all-Wales renal electronic patient database that contains records on all patients in Wales who are under secondary care nephrology follow-up (including prevalent dialysis patients, incident dialysis patients and pre-dialysis patients). For convenience we explain each strand separately below.

\section{Patient and public involvement (co-production)}

The principles of co-production used in this study mean that from inception to dissemination, academics work together with those who provide and use services to improve services through research (figure 4).

To facilitate the co-production we will identify key members of the renal teams and work closely with the WRCN to deliver training via presentations at the National Chronic Kidney Disease meetings and the National Home Therapies meetings. In particular, we will work closely with the clinical nurse specialists based in each of the five renal units across Wales who have a critical role in supporting patient decision-making. We will have detailed conversations with other multidisciplinary team members and clinicians to tell them about the study and to understand more about the ways they work so that we can integrate the research activities appropriately across the five sites. We will also undertake extensive patient and public engagement throughout, using co-production in the study design, set-up and implementation, sharing interim data with key stakeholders - patients, carers, professionals and members of the public with important perspectives.

The co-productive approach will be evaluated through reflections gathered from all those involved in its co-production and framed within the MAGIC approach. The evaluation will be iterative through a systematic, repetitive and recursive process taking place as an integral part of the study based on the guidance for reporting involvement 
How do clinical and socio-demographic factors determine which treatments are discussed and subsequently chosen across Wales?

What do patients and unpaid carers understand about the disease, prognosis and treatment options?

What are the values and preferences of pre and post dialysis patients and their unpaid carers?

Are values and preferences being elicited by clinical teams and how do they impact on treatment choices?

How does economic deprivation, frailty and level of social support influence patient preferences and expectations?

Does clinician bias influence treatment choices and explain some of the regional variation in therapy?

How might we use greater patient involvement to inform better dialysis modality choices?

What are the cost and patient outcome implications of different models of service delivery to support evidence-based policy in renal care across Wales?

How appropriate is the ASCOT measure of social care related quality of life for patients with kidney disease receiving dialysis in different settings?

Would transferring resources between the health sector and the social care sector increase the uptake of home therapies?

How sustainable are current and proposed service models based on evidence from a co-productive study?

Figure 2 Research questions. ASCOT, Adult Social Care Outcomes Toolkit.

of patients and public (GRIPP2) international guidance for reporting of patient and public involvement in health and social care research. ${ }^{22}$

Documentary analysis of current patient education materials and practices

Documents, resources and information on how education is delivered to patients and carers will be collected from each site. Using relevant concepts from the MAGIC
As a co-productive study, patients, service users, and/or their carers are actively involved in this study, particularly in:

Design of the research

Acceptability of the research

Management of the research

Undertaking the research

Analysis of results

Presentation of findings

Figure 4 Co-production.

framework, we will undertake a comparative content analysis to identify and compare the topics and content, mode of delivery and range of resources used in renal units across Wales. Using NVivo qualitative data software, we will deploy an inductive content ${ }^{23}$ analysis approach to assessing current education programme materials and practices, and decision-making procedures followed in Welsh renal centres.

\section{Clinical audit data}

The qualitative element will be a cross-sectional study making use of the Secure Anonymised Information Linkage (SAIL) databank to link clinical and socio-demographic data. The databank allows sourcing, accessing, linking and analysing of health and whole population data all within a governed infrastructure that is safe and secure. Renal patient data will be extracted from the electronic patient record, which has already been embedded

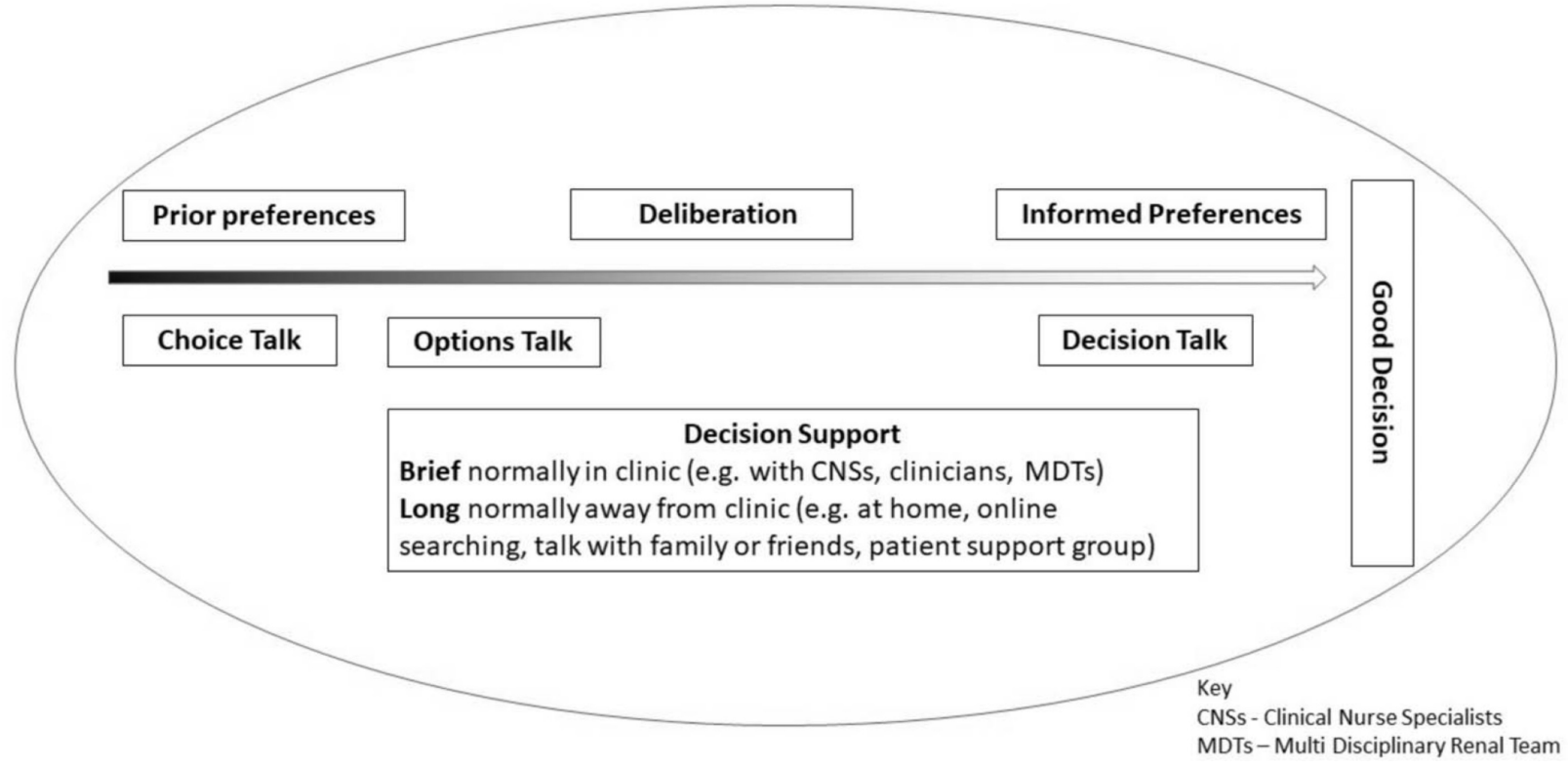

Figure 3 The magic framework. COD, Choices Options and Decisions. 
in SAIL. This will include gender, age, postcode, comorbidity (Charlson comorbidity score), ${ }^{24}$ frailty (Rockwood Clinical Frailty Scale score). Clinical and socio-demographic data will be extracted from the renal electronic patient record, which has been embedded into the SAIL databank. The SAIL databank allows linkage and analysis of pseudo-anonymised routinely collected data in a data safe-haven. The data will include gender, age, postcode, comorbidity (Charlson comorbidity score),${ }^{24}$ frailty (Rockwood Clinical Frailty Scale score), ${ }^{25}$ renal function, quality of life data (EQ-5D-5L score) ${ }^{26}$ Data on the shared decision-making process will be extracted from our all-Wales shared decision-making database which has also been integrated with the SAIL databank. This will include data on which treatments were discussed with patients (and if not why not), which treatments were chosen (and why) and which treatments were not chosen (and why). We will augment this data from the renal electronic patient record by linkage with primary and secondary care data - to identify healthcare utilisation patterns - for instance frequency of hospitalisation, use of primary care services and visits to accident and emergency departments. Analysis will be undertaken using multiple (logistic) regression techniques using SPSS software built into the secure SAIL environment. We will take a $p$ value of $<0.05$ to indicate significance in the absence of multiple comparisons. We will describe $95 \%$ CIs.

\section{Health economics}

We will undertake a rapid review of economic evidence to identify previous published costs of different models of dialysis. Economic specific electronic databases will be searched including; the centre for reviews and dissemination (CRD) Database, NHS economics evaluation database (NHS EED) and health technology assessment (HTA) database. Evidence extracted will be used to inform a budget impact analysis, and a high-level Markov model, ${ }^{27}$ if there is sufficient data to populate the model. The costs of the different dialysis options for the budget impact analysis will also be informed by providers of dialysis treatment in Wales, such as Welsh Health Specialised Services Committee, WRCN and further contacts they suggest. The budget impact analysis will be performed from a NHS and social care perspective.

Cost-consequence analysis is a variant of cost-effectiveness analysis in which an array of consequences/ outcomes (eg, health related quality of life) and costs (eg, health service use costs) are presented, ${ }^{28}$ comparing the two treatment modalities (intervention group vs control group) in a disaggregated form, without combining these into a cost-effectiveness ratio or a cost-utility ratio. ${ }^{29}$ This type of analysis lists the components of an intervention, without making judgements of their relative importance, the verdict is left to the decision maker.

In this cost-consequence analysis, we will present outcomes as the frequency and costs of healthcare use of renal patients gathered from the SAIL databank. In order to answer if different dialysis pathways result in different service use, a cohort of established patients, that is those who have been on their chosen pathway for 3 months or more will be identified from the database. We will focus on the three pathways of; home, unit and peritoneal dialysis. ${ }^{30}$ The time-horizon for the analysis will be 1 year, using a NHS and social care perspective. Information on comorbidities will also be gathered to explore if this has an effect on service use.

To explore the sustainability of current models and how resources could be transferred to the social care sector to increase the uptake of home therapies, health economics specific questions will be added to staff and patient interviews. Staff will be asked about service provision and resources, and the potential for the social sector to support or change current provision. Patients will be asked about productivity losses through changed employment, costs of attending dialysis (eg, transport and parking) and if relevant changes to carer or family member employment if they help the patient to attend dialysis at a hospital or unit (eg, taking annual leave, reducing hours or repaying the time taken by working additional hours at a later date).

\section{Qualitative interviews}

We will interview a minimum of 40 patients and a minimum of 40 unpaid carers from across Wales. Not all patients have unpaid carers so it is likely that not all of the interviews will be with patient/carer dyads. Of the 3000 patients on the electronic record who have made a treatment choice, approximately $20 \%$ to $25 \%$ choose a home therapy $(n=750), 2250$ opting for something else and at least 1 in 5 of these will be undecided (450 approximately). From the total cohort, we will construct a maximum variation sample including from those who were eligible for home therapy and chose a different treatment, or were undecided in the initial sample for interviews across each of the renal units. We will also include patients over 18 who have made a decision and started therapy. Participants will be purposively sampled to ensure a wide range of perspectives and experiences. We can map the overall number with other criteria such as deprivation scale, treatment option, time on dialysis and eligibility for home therapy so we will aim to include additional characteristics of interest in the sample (such as; currently on treatment of choice; men; women; older; younger; multiple comorbidities, socially deprived, etc). Data collection continue until data saturation is reached.

The process for recruiting patients and carers to interview is set out in figure 5 .

Approximately 55 to 60 health and social care professionals currently working in the renal centres are involved in patient decision-making. We will interview a minimum of 20 professionals working across Wales (eg, doctors, nurses, social workers, psychologists, dieticians, occupational therapists, etc) individually and in focus groups. The process to engage and recruit professionals is co-productive: professionals will be working closely with the 


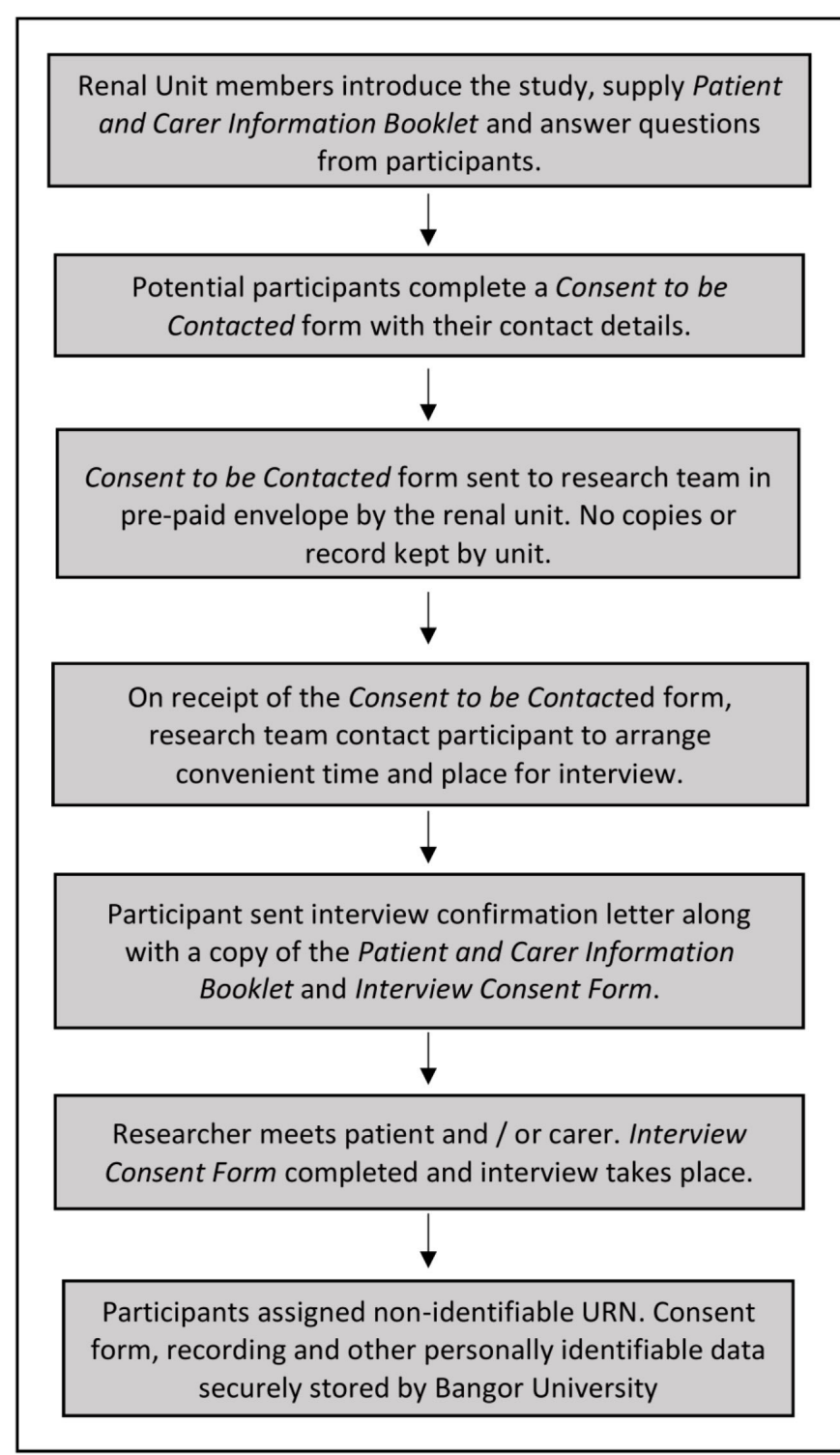

Figure 5 Recruitment process for patients and carers.

research team, the WRCN is a co-partner and the Lead Nurse is a member of the study team.

We will create a sample of the various renal team members that is representative of renal units across Wales. The focus groups and interviews will explore the values, preferences, experiences and expectations and anticipated outcomes from a professional perspective of patient decision-making in making dialysis choices. These focus groups and interviews will also help put emerging findings from the patient and carer interviews into context and contribute to the process of data integration and data analysis.

Digital recordings of interviews will be uploaded into NVivo, classified and coded. Codes will be created to link patients with their unpaid carers and professionals from their respective renal centre. We will use framework analysis to organise and analyse data - using the MAGIC framework as the organising mechanism mapped onto the different treatment pathways and decisions made by patients. ${ }^{19}$

All participants - patients, carers and professionals will be given detailed written information on the study and will be asked to sign a consent form indicating their willingness to participate. Anonymity will be assured to participants. We will expressly ask participants for their anonymised data to be used for all purposes (in this study, to be stored for use in further studies, for teaching and training). Participants retain the right at all times to withdraw from the study at any time and patients will be assured that withdrawal will have no impact on their current or future treatment.

\section{Data integration}

Data integration will occur at a number of levels using the MAGIC framework ${ }^{19}$ and as the underpinning mechanism for integration organised by treatment pathways and decisions. Qualitative data from patients and unpaid carers will for example be used to explain in more nuanced detail the patient and unpaid carer reasoning and explanations underpinning the different treatment decisions recorded in the patient database. Documentary analysis of patient education programmes will for example be used to explain any obvious differences in the way programmes are delivered and any trends in promoting treatment options by centre. Professional interviews will also be used to compare the clinical and patient experiences. Drawing on anonymised cases from the patient interviews, we will develop a small number of scenarios to put to professionals, looking for any evidence of any conscious or unconscious biasses in recommended treatment options that may influence patient decision-making, as well as highlighting any issues for context such as resources and availability of treatments in specific centres. Data from the qualitative study will help inform the economic investigation, for example, whether it may be beneficial shift resources from health to social care and what types of costs these might be.

\section{ETHICS AND DISSEMINATION \\ Ethical issues}

We have considered the following ethical issues in designing this study:

1. Protection of individual medical information. For clinical and economic database analyses, routinely collected clinical data has already been entered into the SAIL databank which is pseudo-anonymised (personally identifiable information fields within a data record are replaced by one or more artificial identifiers or pseudonyms). In this study, we will extract aggregate data for our analysis - so no patient identifiable data is held.

2. Protection of identifiable participant information. Only the minimum information necessary to contact and interview participants will be collected.

3. Participant consent to be interviewed. Eligible patients will be invited to enter the study by renal unit 
multidisciplinary team members who are known to the patients. We will use standardised protocols to ensure that patients are identified sensitively and given information in a suitable way and at a suitable time. Prior to interview, patients and carers will be fully consented. At each stage of the recruitment process (figure 5) participants will have the opportunity to ask questions, consider their participation or withdraw with no consequences. We have protocols in place for supporting field researchers including lone worker policies.

4. There is the possibility of patients and carers sharing details which might cast light on the nurse and clinical practices, therefore the patient interviews will be anonymised, and data shared back in a way that will not identify either patients, carers or professionals.

5. There is a small possibility that the clinical teams will feel that their practices are under unwanted scrutiny, we will work with the renal teams throughout to build good working relationships and positively encourage the teams to share any concerns with us.

6. Full interview safeguards and protocols will be put in place to ensure that there is no undue pressures on the patients and carers during interview. Participants will be offered a courtesy follow-up call and the research team will disengage.

7. Any serious concerns for the safety of any participants will be processed following the safeguarding policy in the respective organisation who provides care for the person. We will make this point clear in the consent process.

8. Data management. There are multiple organisations working co-productively on this study. We have designed the study and developed specific General Data Protection Regulation compliant protocols to ethically and safely collect and store data and to ensure that no personally identifiable data is shared between parties.

\section{Dissemination and impact}

As a co-productive study involving patients, clinicians, third sector partners and academics, findings from this study will be shared on a continual basis. Responses from partners to findings are an intrinsic part of the evolution of co-production research. Our collaboration with the WRCN, means that we are able to rapidly disseminate our findings and influence patient care and service commissioning. We speculate that the findings of our study may lead to the following changes:

- Incorrect patient assumptions about disease/therapy/ prognosis: If we find that patient's perception of their disease and illness trajectory differs from their clinician's views then we will use the WRCN to overhaul the current pre-dialysis education programme. It is likely that the delivered information (and the way in which it is delivered) needs to be changed. This may include giving information earlier (and more gradually) as well as the use of multimedia technology. We will use the patient and carer interviews to help develop an education process that better elicits patients' preferences and values so they can be matched to treatment

- Lack of carer/support network: If we find that social isolation is a factor that determines treatment choice, then together with the WRCN we will explore the role of utilising the social care sector for example, transferring costs from the healthcare sector to paid carers (this may form the basis of a future health economic study).

- Accommodation problems/concerns about cost: Lack of appropriate housing may prove to be one of the factors that limits treatment choice in Wales. In regions where this is the case we will look at the role of third sector in supporting patients to access benefits/re-housing (eg, employing benefits support officers). The WRCN has previously worked alongside third sector organisations to provide a youth worker to support younger patients come to terms with their illness and it may be that we need to follow the same model to support vulnerable older patients. Such an approach is likely to prove significantly cost-effective if it leads to more patients choosing to have dialysis at home.

- Variation between clinical teams regarding patient selection for home therapy: If we confirm significant variation in care between clinical teams, then (via the established quality and patient safety process) the WRCN will develop robust guidelines for clinical teams with standardised criteria defining which clinical conditions preclude a home therapy. Given that the unique position that the WRCN holds as a clinically led commissioning network with a track record of rapid service change, we feel that it is entirely feasible to expect that the findings of our study can rapidly change practice and improve patients care across Wales.

\section{Author affiliations}

${ }^{1}$ Cardiff and Vale NHS Trust, Cardiff, UK

${ }^{2}$ Renal Unit, Abertawe Bro Morgannwg University Health Board, Swansea, UK

${ }^{3}$ Patient and public representative, Swansea, UK

${ }^{4}$ School of Health Sciences, Bangor University, Bangor, UK

${ }^{5}$ Wales Renal Clinical Network, Swansea, UK

${ }^{6}$ Centre for Health Economics \& Medicines Evaluation, Bangor University, Bangor, UK

${ }^{7}$ School of Health Sciences, Bangor University College of Health and Behavioural

Sciences, Bangor, UK

Acknowledgements The authors would like to acknowledge the wider network of public and professionals who helped to develop this study through the Wales Kidney Research Unit.

Contributors GR, JN, JC, LM, and RE were responsible for the conceptual design of the study and funding application. GR, JN, JC, DD, TH, GW, LM JMC and RE were responsible for developing and further operationalising the methodology and theoretical underpinning. GR, JC, JMC, LM, DD and JN drafted the initial manuscript. All authors revised and approved the final manuscript with the exception of TH who sadly died before submission.

Funding This work was funded by Health and Care Research Wales (HCRW). The views expressed are those of the authors and not necessarily those of NHS Wales and HCRW.

Competing interests None declared. 
Patient consent for publication Not required.

Ethics approval The study has full approval from Health and Care Research Wales Research Ethics Committee \#5.

Provenance and peer review Not commissioned; externally peer reviewed.

Data availability statement № data are available.

Open access This is an open access article distributed in accordance with the Creative Commons Attribution Non Commercial (CC BY-NC 4.0) license, which permits others to distribute, remix, adapt, build upon this work non-commercially, and license their derivative works on different terms, provided the original work is properly cited, appropriate credit is given, any changes made indicated, and the use is non-commercial. See: http://creativecommons.org/licenses/by-nc/4.0/.

\section{ORCID iDs}

Gareth Roberts http://orcid.org/0000-0003-4496-6539

D J Dallimore http://orcid.org/0000-0002-4557-8186

Jane Noyes http://orcid.org/0000-0003-4238-5984

\section{REFERENCES}

1 Kidney Care UK. Facts and Stats. A range of useful facts and stats about kidneys. Available: https://www.kidneycareuk.org/news-andcampaigns/facts-and-stats/ [Accessed 19 Mar 2019].

2 Kerr M, Bray B, Medcalf J, et al. Estimating the financial cost of chronic kidney disease to the NHS in England. Nephrol Dial Transplant 2012;27(suppl_3):iii73-80.

3 National Institute for Health and Care Excellence (NICE). Chronic kidney disease (partial update). early identification and management of chronic kidney disease in adults in primary and secondary care, 2014. Available: https://www.nice.org.uk/guidance/cg182/evidence/ full-guideline-pdf-191905165 [Accessed 26 Mar 2019].

4 Welsh Renal Clinical Network. Renal services in Wales 2016 - 2020 delivery plan, 2016. Available: http://www.wales.nhs.uk/sites3/ Documents/773/Renal Disease Quality Delivery Plan1.pdf [Accessed 10 Apr 2019].

5 Macneill SJ, Ford D, Evans K, et al. Uk renal registry 20th annual report: Chapter 2 UK renal replacement therapy adult prevalence in 2016: national and Centre-specific analyses. Nephron 2018;139:47-74.

6 Morton RL, Tong A, Howard K, et al. The views of patients and carers in treatment decision making for chronic kidney disease: systematic review and thematic synthesis of qualitative studies. $B M J$ 2010;340:c112-3.

7 Ormandy P. Information topics important to chronic kidney disease patients: a systematic review. J Ren Care 2008;34:19-27.

8 Walker RC, Howard K, Morton RL, et al. Patient and caregiver values, beliefs and experiences when considering home dialysis as a treatment option: a semi-structured interview study. Nephrol. Dial. Transplant. 2016;31:133-41.

9 Walker RC, Morton RL, Palmer SC, et al. A discrete choice study of patient preferences for dialysis modalities. Clin J Am Soc Nephrol 2018;13:100-8.
10 Walker RC, Howard K, Tong A, et al. The economic considerations of patients and caregivers in choice of dialysis modality. Hemodial Int 2016;20:634-42.

11 Evans RW, Manninen DL, Garrison LP, et al. The quality of life of patients with end-stage renal disease. N Engl J Med 1985;312:553-9.

12 Oberley ET, Schatell DR. Home hemodialysis: survival, quality of life, and rehabilitation. Adv Ren Replace Ther 1996;3:147-53.

13 Berger JR, Hedayati SS. Renal replacement therapy in the elderly population. Clin J Am Soc Nephrol 2012;7:1039-46.

14 Verberne WR, Geers ABMT, Jellema WT, et al. Comparative survival among older adults with advanced kidney disease managed conservatively versus with dialysis. Clin J Am Soc Nephrol 2016;11:633-40.

15 Walker RC, Walker S, Morton RL, et al. Māori patients' experiences and perspectives of chronic kidney disease: a new Zealand qualitative interview study. BMJ Open 2017;7:e013829.

16 National Institute for Clinical Excellence (NICE). Renal failure-home versus Hospital haemodialysis, 2011. Available: https://www.nice. org.uk/guidance/ta48 [Accessed 19 Mar 2019].

17 National Institute for Clinical Excellence (NICE). Bringing the benefits of home haemodialysis home. Shar. learn. database, 2011. Available: https://www.nice.org.uk/sharedlearning/bringing-the-benefits-ofhome-haemodialysis-home [Accessed 19 Mar 2019].

18 Chertow GM, Levin NW, Beck GJ, et al. Long-Term effects of frequent In-Center hemodialysis. J Am Soc Nephrol 2016;27:1830-6.

19 Joseph-Williams N, Lloyd A, Edwards A, et al. Implementing shared decision making in the NHS: lessons from the magic programme. BMJ 2017;357:j1744.

20 Elwyn G, Frosch D, Thomson R, et al. Shared decision making: a model for clinical practice. J Gen Intern Med 2012;27:1361-7.

21 King E. Evaluation of making good decisions in collaboration (magic) programme; 2013: 1-173. http://www.unfpa.org/sites/default/files/ admin-resource/BoliviaReport1_EN_2.pdf

22 Staniszewska S, Brett J, Simera I, et al. GRIPP2 reporting checklists: tools to improve reporting of patient and public involvement in research. BMJ 2017;358:j3453.

23 Krippendorff K. Content analysis: an introduction to its methodology. Third ed. London: Sage Publications, 2012.

24 Charlson M, Szatrowski TP, Peterson J, et al. Validation of a combined comorbidity index. J Clin Epidemiol 1994;47:1245-51.

25 Rockwood K, Andrew M, Mitnitski A. A comparison of two approaches to measuring frailty in elderly people. J Gerontol A Biol Sci Med Sci 2007;62:738-43.

26 Rabin R, Charro Fde. EQ-SD: a measure of health status from the EuroQol group. Ann Med 2001;33:337-43.

27 Drummond MMF, Sculpher MMJ, Claxton K, et al. Methods for the economic evaluation of health care programmes. Oxford University Press: Oxford:, 2015

28 Glick H, Doshi J, Sonnad S, et al. Economic evaluation in clinical trials. Oxford University Press: Oxford:, 2015.

29 Brazier J, Ratcliffe J, Saloman J, et al. Measuring and valuing health benefits for economic evaluation. Oxford University Press: Oxford:, 2017.

30 Personal Social Services Research Unit. Adult social care outcomes toolkit. Univ. Kent, 2018. Available: https://www.pssru.ac.uk/ascot/ tools/ [Accessed 19 Mar 2019]. 